\title{
The de novo sequence origin of two long non-coding genes from an inter-genic region
}

\author{
Yulin Dai ${ }^{1,2+}$, Shengdi Li ${ }^{1,2+}$, Xiao Dong ${ }^{1,2}$, Han Sun ${ }^{1,2,3}$, Chao Li ${ }^{1,2}$, Zhi Liu ${ }^{1,2}$, Beili Ying ${ }^{4}$, Guohui Ding ${ }^{1,3^{*}}$, Yixue Li ${ }^{1,3^{*}}$ \\ From The International Conference on Intelligent Biology and Medicine (ICIBM 2013) \\ Nashville, TN, USA. 11-13 August 2013
}

\begin{abstract}
Background: The gene Polymorphic derived intron-containing, known as Pldi, is a long non-coding RNA (IncRNA) first discovered in mouse. Although parts of its sequence were reported to be conserved in rat and human, it can only be expressed in mouse testis with a mouse-specific transcription start site. The consensus sequence of Pldi is also part of an antisense transcript AK158810 expressed in a wide range of mouse tissues.

Result: We focused on sequence origin of Pldi and Ak158810. We demonstrated that their sequence was originated from an inter-genic region and is only presented in mammalians. Transposable events and chromosome rearrangements were involved in the evolution of ancestral sequence. Moreover, we discovered high conservation in part of this region was correlated with chromosome rearrangements, CpG demethylation and transcriptional factor binding motif. These results demonstrated that multiple factors contributed to the sequence origin of Pldi.
\end{abstract}

Conclusions: We comprehensively analyzed the sequence origin of Pldi-Ak158810 loci. We provided various factors, including rearrangement, transposable elements, contributed to the formation of the sequence.

\section{Introduction}

Although pervasively transcribed, only $5 \%-10 \%$ of the human genome is covered by mRNA and spliced noncoding RNAs, and the majority of which does not encode proteins [1]. Long non-coding RNAs (lncRNAs) are defined as transcribed non-coding RNA larger than $200 \mathrm{nt}$ in length, which plays an essential role in regulating gene expression, chromatin functions [2]. As lncRNAs act as biological building blocks, it is necessary to understand the process of developing new lncRNA genes [3].

The emergence of a functional lncRNA gene could be summarized into various evolutionary scenarios, including metamorphosis of a protein coding gene, derived from a genomic region previously devoid of exonic sequence, duplication by retro-transposition, and emergence following tandem duplication or insertion of

\footnotetext{
*Correspondence: gwding@sibs.ac.cn; yxli@sibs.ac.cn

+ Contributed equally

${ }^{1}$ Key Laboratory of Systems Biology, Shanghai Institutes for Biological

Sciences, Chinese Academy of Sciences, 320 Yueyang Rd. Shanghai 200031, PR China

Full list of author information is available at the end of the article
}

transposable elements [1,4]. For most of the scenarios, comprehensive studies have been established on specific lncRNA genes with well-known functions, such as XIST, HOTAIR [5,6]. However, little was known about developing a new lncRNA gene from a non transcribed genomic region. The mechanism of the de novo origin of a lncRNA gene remains to be clarified.

Previous study on de novo protein has accounted for that those seemingly dispensable sequences in non-genic regions could generate adaptive functional proteins through evolution. The de novo birth and development of a potential protein coding gene is in line with increasing open reading frame (ORF) length and conservation through the natural selection benefited from random translation on genome $[3,7,8]$. Like proteins, the occasional transcription and changing events in non-genic sequences could provide raw material generating de novo lncRNAs [9]. Here, we focused on the sequence origin of a lncRNA in an intergenic region, demonstrating its sequence components and changes within species.

Pldi gene was previously identified and defined as an intergenic originated lncRNA gene, which is overlapped 
with a putative opposite-strand transcript, $A K 158810$ (Additional File 1). Pldi locates within a $200 \mathrm{~kb}$ region that is free of annotated transcripts or expressed sequence tags (ESTs) in rat and humans, which raise the possibility of de novo emergence of the Pldi-AK158810 loci (about 20 kbps-long). Knocking out Pldi would reduce sperm motility and testis weight, indicating that Pldi has the ability in regulating the expression levels of other genes in testis [10]. Numerous functional noncoding RNAs have been demonstrated to regulate gene expressions through an antisense mechanism, playing an important role of gene overlapping in non-coding RNA functions [11-13]. On the contrary, few studies discussed the origin of overlapping non-coding RNAs due to lacking of clear markers, like ORF in protein.

In this study, we conducted a comprehensive analysis on the sequence origin of mouse Pldi-Ak158810 loci. We evaluated various factors that contribute to the origin, and gave adequate evidence to prove the de novo origin of this loci. Moreover, we found that PldiAk158810 established its fixation from a specific overlapping region some time before emergence. We further discussed the potential role of the local element in the evolution and fixation of this orphan lncRNA gene loci.

\section{Materials and methods}

\section{Genomes and sequences}

The 13 genomes of vertebrates used in this study were downloaded from UCSC genome database http://hgdownload.soe.ucsc.edu/downloads.html. Genome versions of these 13 genomes are in Additional File 2. The sequence of Pldi-Ak158810 loci was picked from mouse (GRCm 38) export data in Ensembl http://www.ensembl.org.

\section{ORF analysis}

Sequences of EST Ak158810 were checked to find all the potential open reading frames, by using ORF finder (Open Reading Frame Finder) by default minimum frame size. The ORF finder is accessible in this website server http://www.ncbi.nlm.nih.gov/gorf/gorf.html[14]

\section{Sequence comparison and alignment, phylogeny analysis} We used nucleotide Blast (Basic Local Alignment Search Tool) to detect homology between Pldi-Ak158810 nucleotide sequence and vertebrate genomes, a cutoff for identity was set at $80 \%$. Protein Blast was used to find protein coding genes homologue to the genes flanking with Pldi and Ak15880. ClustalW http://www.clustal. org/download/current/ was used to align protein and nucleotide sequences [15]. MEGA5.1 was used to construct neighbor-joining phylogenic tree [16]. The genomic alignment of 30 vertebrates by MultiZ was downloaded from UCSC $[17,18]$. All genomes were mapped to the mouse chromosomes.

\section{Repeats and transposable elements annotation}

Repeats and transposable elements were annotated by Repeatmasker program. Sequences of Pldi-Ak158810 were submitted to the Repeatmasker website http://www. repeatmasker.org version 4.0.1, which uses default parameters. The repeat class were transformed and grouped as SINE, LINE, DNA, LTR and others. In the analysis of ancient transposable elements, we did not include simple repeats and low complexity sequences [19].

\section{Model for substitution rate change}

A simple model was constructed to test the substitution rate change relative to exons of surrounding genes. Three constant substitution rates were defined as: $r_{0}$, rate before inversion; $r_{1}$, rate after inversion and before gene birth; $r_{2}$, rate after gene birth. As surrounding genes' reference sequence, the rates were summarized as $\mathrm{r}_{\mathrm{R}}$ encompassing the whole phylogenetic tree. We assumed the substitution rates relative to those exons will not change significantly if no selection pressures affect this region. A time interval was estimated instead of an exact time point, of the inversion and gene birth, so we used two variables $k_{1}, k_{2}$, ranging from $0 \sim 1$ to reflect the timing of two events. $k_{1}$ is the proportion of the time from present to the common ancestor of human and rat, in which the inversion has occurred. $\mathrm{k}_{2}$ is the proportion from present to the common ancestor of mouse and rat, for the emergence of lncRNA transcription. The distance between species $X$ and $Y d_{X Y}$ can be approximately calculated as $d_{X Y}=2 \cdot r_{X Y} \cdot t_{X Y}$, if the rate is regarded as constant. Under the assumptions of our model (Figure 1), for test sequence we get ():

$$
\begin{aligned}
& \mathrm{d}^{\text {test }}{ }_{\mathrm{HD}}=2 \cdot \mathrm{r}_{0} \cdot \mathrm{t}_{\mathrm{HD}} \\
& \mathrm{d}^{\text {test }}{ }_{\mathrm{HR}}=\left(1+\mathrm{k}_{1}\right) \cdot \mathrm{r}_{0} \cdot \mathrm{t}_{\mathrm{HR}}+\left(1-\mathrm{k}_{1}\right) \cdot \mathrm{r}_{1} \cdot \mathrm{t}_{\mathrm{HR}}, \\
& \mathrm{d}^{\text {test }}{ }_{\mathrm{MR}}=\left(1+\mathrm{k}_{2}\right) \cdot \mathrm{r}_{1} \cdot \mathrm{t}_{\mathrm{MR}}+\left(1-\mathrm{k}_{2}\right) \cdot \mathrm{r}_{2} \cdot \mathrm{t}_{\mathrm{MR}},
\end{aligned}
$$

where $\mathrm{d}_{\mathrm{HD}}^{\text {test }}, \mathrm{d}_{\mathrm{HR}}^{\text {test }}, \mathrm{d}^{\text {test }}{ }_{M R}$ are the genetic distance of the test sequences between human and dog, human and rat, mouse and rat, respectively, $t_{\mathrm{HD}}, \mathrm{t}_{\mathrm{HR}}, \mathrm{t}_{\mathrm{MR}}$ represent for the divergent time between each pair of species.

For reference sequence,

$$
\begin{aligned}
& d_{H D}^{\text {Ref }}=2 \cdot r_{R} \cdot t_{H D}, \\
& d^{\text {Ref }}{ }_{H R}=2 \cdot r_{R} \cdot t_{H R}, \\
& d^{\text {Ref }}{ }_{M R}=2 \cdot r_{R} \cdot t_{M R},
\end{aligned}
$$

where $\mathrm{d}_{\mathrm{HD}}^{\mathrm{Ref}}, \mathrm{d}_{\mathrm{HR}}^{\mathrm{Ref}}$, and $\mathrm{d}_{\mathrm{MR}}^{\mathrm{Ref}}$ are the genetic distance of the reference exon sequences between human and dog, human and rat, mouse and rat. 
a)

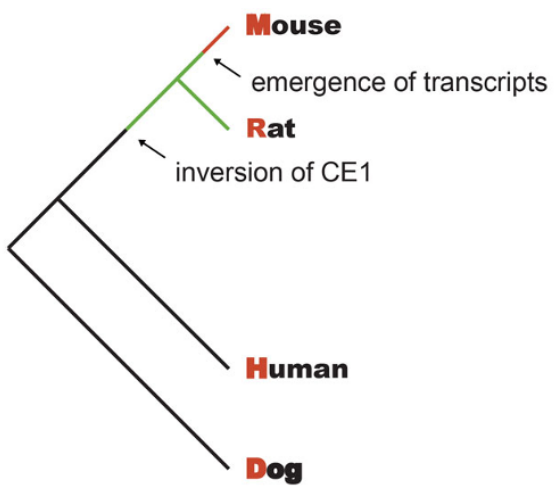

c)

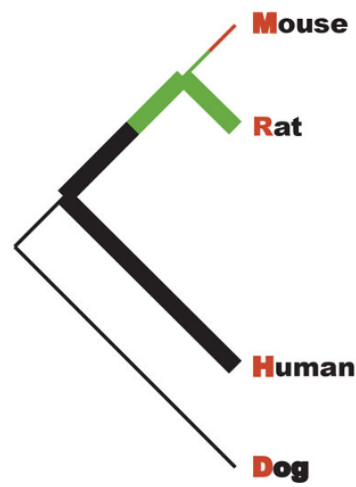

b)

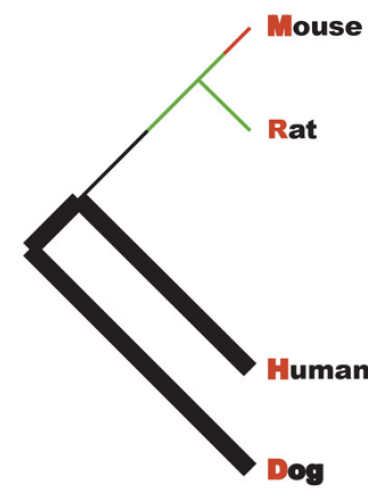

d)

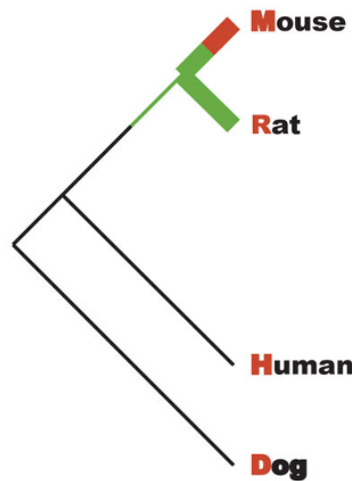

Figure 1 Assumptions of the model. We used surrounding gene exons as reference. We assumed the substitution rate relative to those exons would not change too much if no selection pressures affect this region. For test sequence, we defined three constant substitution rates on the species tree, to test the rate change at two time point: inversion and emergence of Pldi-Ak158810 transcription. (a) Red lines represent for substitutions after gene birth, at rate $r_{2}$; green lines represent for substitutions after two observed inversion, and before gene birth, at rate $r_{1}$; black lines represent for substitutions before inversion, at rate $r_{0}$. We were concerned about the rate change relative to surrounding gene exons, all the rates were normalized by the variable reference rate $r_{R}$. $(b-d) r_{\text {averXY }}$ were calculated by dividing test sequence $X Y$ distance by reference $X Y$ distance, which indicates a relative substitution rate on the bold path; three $r_{a v e r X Y}$ will be similar if substitution rates do not vary significantly in three stages.

We calculated three average substitution rates,

$$
\begin{aligned}
& \mathrm{r}_{\text {averHD }}=\mathrm{d}^{\text {test }}{ }_{\mathrm{HD}} / \mathrm{d}^{\text {Ref }}{ }_{\mathrm{HD}}=\mathrm{r}_{0} / \mathrm{r}_{\mathrm{R}}, \\
& \mathrm{r}_{\text {averHR }}=\mathrm{d}^{\text {test }}{ }_{\mathrm{HR}} / \mathrm{d}^{\text {Ref }}{ }_{\mathrm{HR}}=\left[\left(1+\mathrm{k}_{1}\right) \cdot \mathrm{r}_{0}+\left(1-\mathrm{k}_{1}\right) \cdot \mathrm{r}_{1}\right] / \mathrm{r}_{\mathrm{R}} \\
& \mathrm{r}_{\text {averMR }}=\mathrm{d}_{\mathrm{MR}}^{\text {test }} / \mathrm{d}_{\mathrm{MR}}^{\text {Ref }}=\left[\left(1+\mathrm{k}_{2}\right) \cdot \mathrm{r}_{1}+\left(1-\mathrm{k}_{2}\right) \cdot \mathrm{r}_{2}\right] / \mathrm{r}_{\mathrm{R}},
\end{aligned}
$$

where $0<\mathrm{k}_{1}, \mathrm{k}_{2}<1 . \mathrm{r}_{\text {averHD}}, \mathrm{r}_{\text {averHR }}$ and $\mathrm{r}_{\text {averMR }}$ are the average substitution rates at different stages. If $r_{\text {averHR }}<$ $(>) r_{\text {averHR }}$, we get $r_{0}<(>) r_{1}$. Similarly, a reduced $r_{2}$ will produce a lower $r_{\text {averMR }}$, as the only envolving path affected by $r_{2}$.

We used ClustalW to realign the conserved elements in Pldi-Ak158810 and exons of surrounding four genes, manually remove sites with low similarity by Bioedit http://www.mbio.ncsu.edu/bioedit/bioedit.html. All the four genes were merged into one single alignment. Then a Maximum Likelihood (ML) tree and distance matrix was estimated by PAML 4.6 baseml for each alignment) [20].

\section{Methylations data}

We collected two sources of methylation data as a comparison, one is from mouse tissue, the other is from human ENCODE data.

Mouse brain methylation data was obtained from forebrain tissue of lab mouse (GSM809309)

The probability of methylation was estimated with both methylated and unmethylated fragment information (Additional File 3) [21].

Demethylation data from human UCSF brain methylation database viewed with UCSC genome browser was implemented to detect the DNA methylation in the human homologue region of Pldi-Ak158810 loci, which was displaced in Additional file 3[22].

\section{RNA-seq data}

RNA-seq data is from Encode Cold Spring Harbor Lab (CSHL) RNA-seq, and there are 5 types of tissues included (heart, kidney, ovary, spleen and testis). We viewed this data using UCSC genome browser [23]. 


\section{Transcriptional factor binding site data}

Human, Mouse, Rat (HMR) Conserved Transcriptional Factor Binding Site (TFBS) was implemented to displace the potential binding sites of these two highly conserved regions [24].

http://www.biobase-international.com/library/transfac

\section{Results}

The co-emergence of PIdi and Ak158810 transcription

We studied the emergence time of Pldi and Ak158810. $P l d i$ locates in an inter-genic region free of any human and rat EST signals, indicating that Pldi and its antisense putative gene generating $A k 158810$ were not transcribed before the divergence of rodents. In mouse lineages, RNA transcript of Pldi has been discovered [10]. To validate the transcript Ak158810, we compared its $2.9 \mathrm{~kb}$ sequence with mouse EST database from NCBI. The result confirmed the transcription of Pldi antisense strand, and EST hits matched with splicing of the first and second exons of Ak158810 (Additional File 4). We further analyzed the open reading frames (ORFs) in Ak158810 RNA, The longest ORF is shorter than 110 amino acids. (Two AUG codons with shorter reading frames $\sim 70$ amino acids preceded this long ORF) (Additional File 5). It indicates that $A k 158810$ is not likely to encode proteins. Our results, along with previous knowledge [10], showed that the Pldi and $A k 158810$ are two mouse-specific lncRNAs located on anti-sense strand to each other. These evidences suggest that Pldi, and its putative antisense lncRNA, Ak158810, were first transcribed at similar time between the divergence of mouse and rat.

\section{Pldi-Ak158810 loci is conserved in mammals and originated from an intergenic region}

To study the evolution of Pldi-Ak158810 loci, we searched for homologues of Pldi and Ak158810 loci in 13 vertebrates. First, homologs of Pldi-Ak158810 sequence were found in all mammals by using Blastn. Except a transposable element in rat and mouse, all the homologs are between the region of unc $5 \mathrm{~b}$ and pcbd1 in mammalian classes. It demonstrates that parts of Pldi-Ak158810 loci were already present in the mammalian cen-ancestor. Meanwhile, we failed to detect significant sequence similarity to Pldi-Ak158810 loci in nonmammalian vertebrates with Blastn (Figure 2 \& Additional File 6).
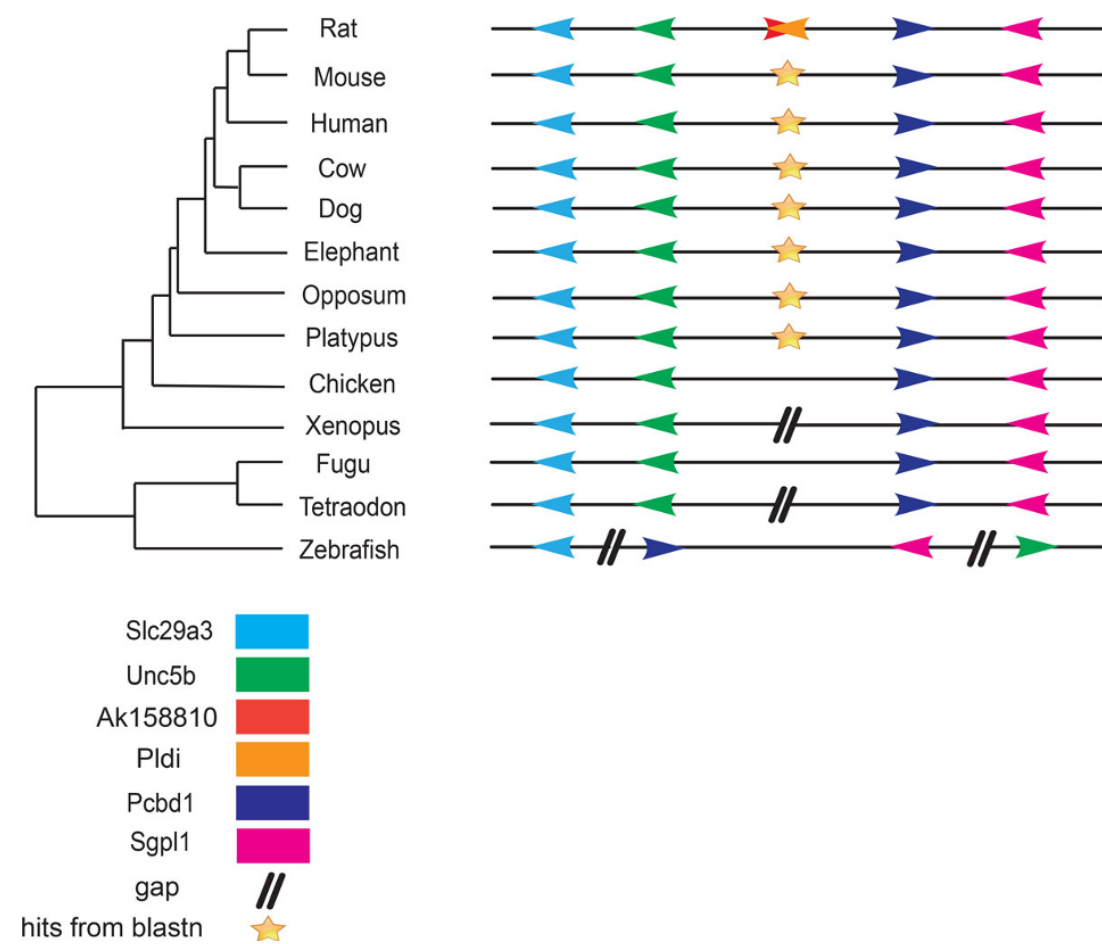

Figure 2 Phylogenetic distribution of PIdi-Ak158810 loci and its surrounding genes within vertebrate species. The phylogenetic tree of 13 vertebrates was adapted from a widely accepted tree topology [5,32]. The branch length does not represent the distance between each species and no molecular clock model was assumed. Different gene highlighted with marks of different colors. We could find the hits from Blastn in all mammals, whereas no hits in non-mammalian species. We could observe that all these 4 flanking protein are ordered laid around the Pldi-Ak158810 loci. In contrast, in non-mammalian species, some big gaps (larger than the average distance $200 \mathrm{~kb}$ in mammals) inserted into the 4 flanking protein region, which made the order of the 4 proteins changed. The gap in Xenopus might due to the incomplete genomic description. Result of Blastn could be found in Additional File 6. 
In mouse, a $\sim 450 \mathrm{~kb}$ genomic region surrounding Pldi and Ak158810, contains four protein coding genes (slc29a3, unc5b, pcbd1 and sgpl1) that have orthologous genes in vertebrates (Figure 2 \& Figure 3). In 12 vertebrates, the gene content, order and orientation of four flanking genes are perfectly conserved. In zebrafish, the linkage of the four genes is broken (Figure 2). However, this does not correspond to the ancestral state of non-tetrapod vertebrates, for it is the only mismatch among three kinds of fish (fugu, tetraodon, zebrafish). These results denoted that the genomic region where Pldi and Ak158810 emerged is stable since the cen-ancester of vertebrates, which indicated that PldiAk158810 loci originated from an intergenic region linkaged in non-tetrapod vertebrates and remained conservation in mammals

\section{The origins of exons and introns}

Based on the exons and introns of Pldi and Ak158810, we further analyzed the origin of them in mouse, rat, human, dog, opossum and chicken (Figure 4 \& Figure 5). Pldi consists of 3 exons and 2 introns, and Ak158810 consists of 5 exons and 4 introns. In non-rodent species, two fragments in intron 1 of Pldi were detected. Then, we used MultiZ alignment to compare Pldi and its homologues regions in mouse, rat, human, dog, chicken and opossum. We discovered that the majority of the mouse Pldi-Ak158810 region could be aligned to rat, human, and dog, including exons and introns. In opossum, no fragment was mapped to the three Pldi exons. $A k 158810$ exon 1 and part of exon 3 are covered by opossum homologues, matching with the conserved elements detected by our previous analysis (Figure 2). In chicken, few homologue was detected, except partial Ak158810 exon 3.

The sequence alignment also revealed that were involved in the evolution of Pldi region. We identified two inversions at the loci. First one is the inversion of a $\sim 800$ bps fragment, containing the first exon of Ak158810 (Figure 5 \& Addition File 1). Another inversion is overlapped with $A k 158810$ exon 5. The regions, homologue to the two inversed fragments of non-rodent mammalians, are in opposite direction to those of mouse, which reveals both inversions occurred before the divergence of mouse and rat, and after the divergence of primates and rodents. Interestingly, the first inversed region is highly conserved, which is discussed in following section.

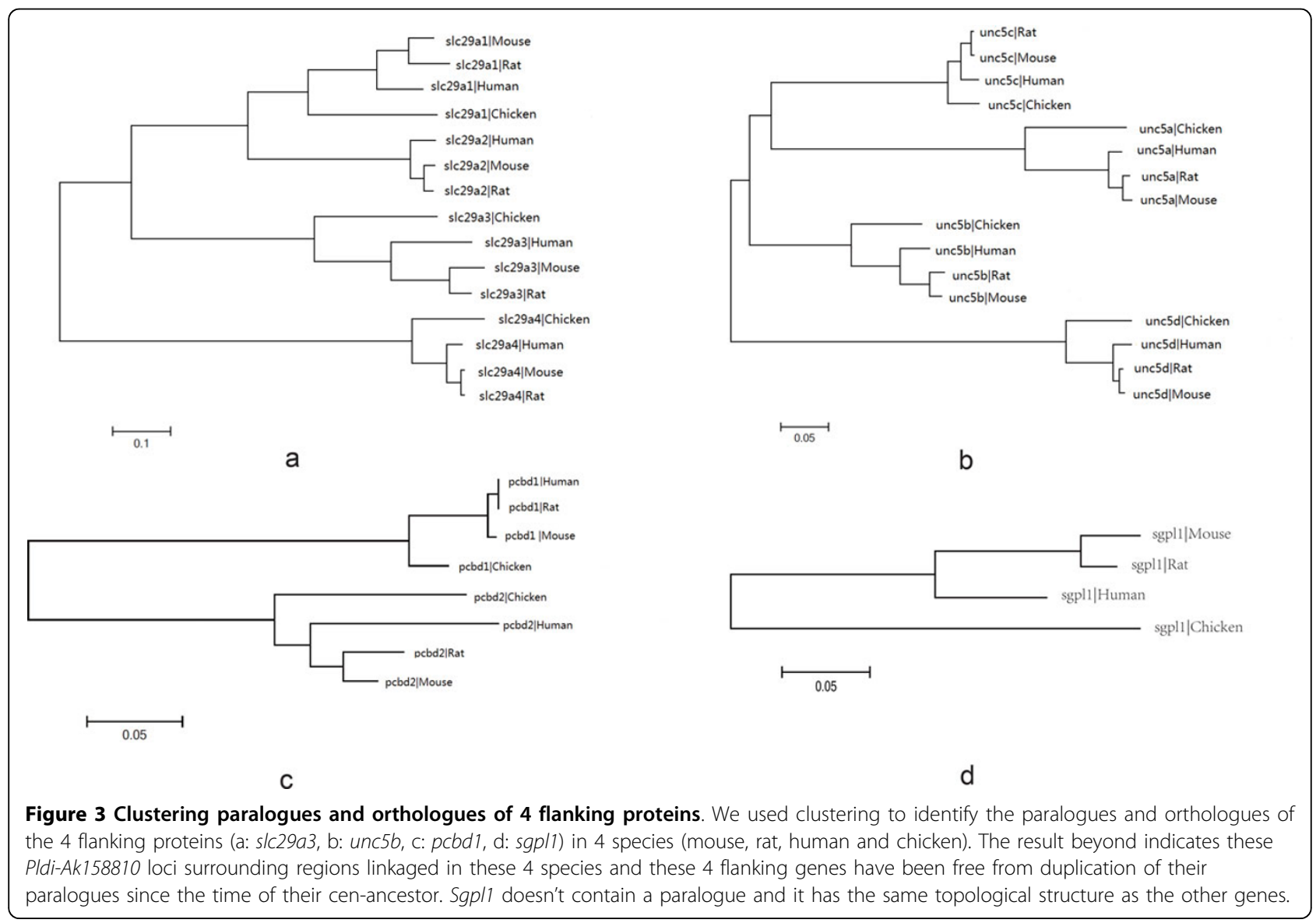




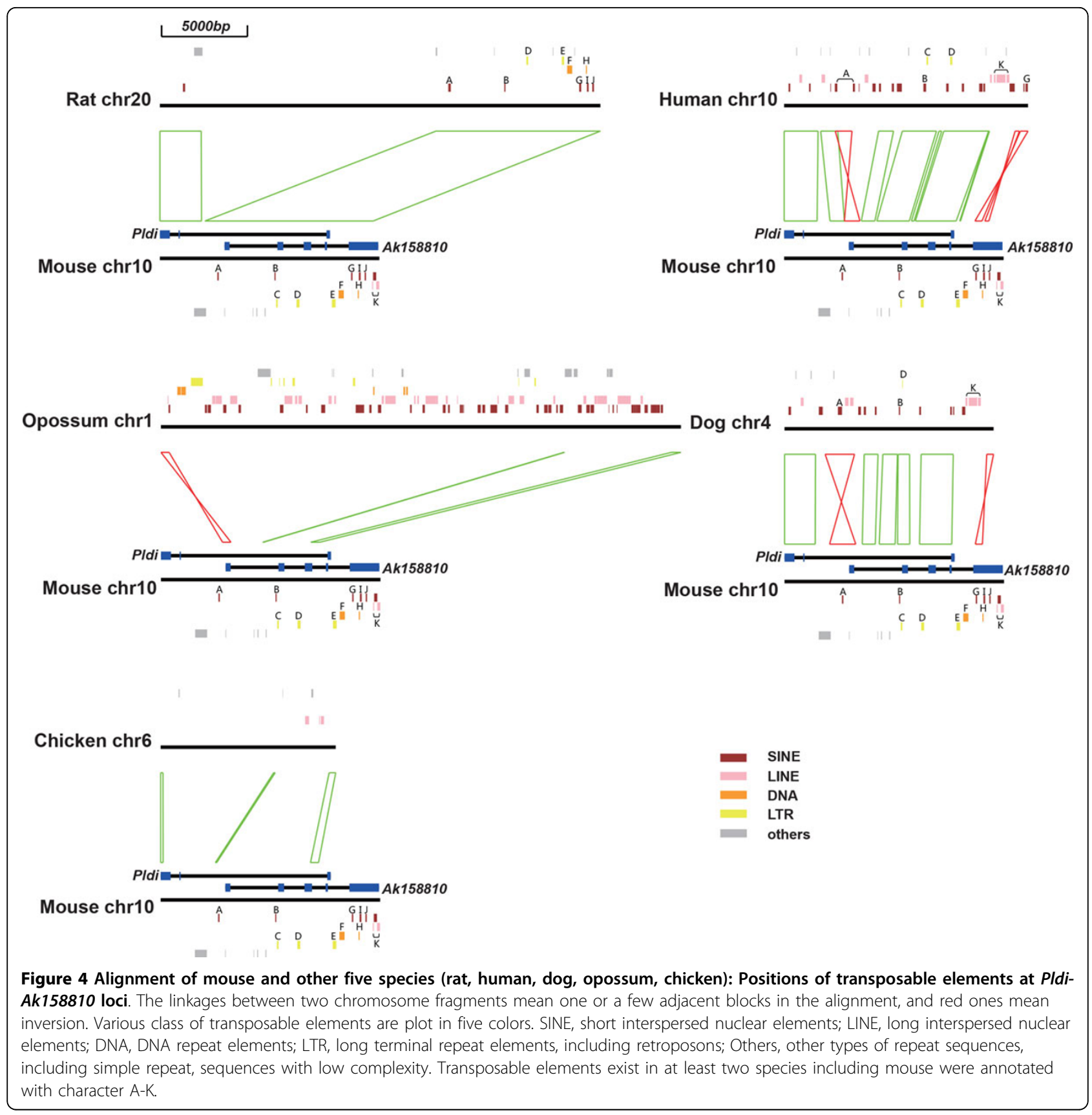

Transposable events contribute to the formation of PldiAk158810 loci

Transposable elements (TEs) have been considered as an important composition in the genome [25], we then evaluated the contribution of transposons to the formation of Pldi-Ak158810 loci. We compared the sequence of mouse Pldi-Ak158810 loci and homologous sequences in other 5 species (rat, human, dog, opossum and chicken) with the database of mobile elements, using RepeatMasker program [19].To understand whether Pldi-Ak158810 loci is interrupted by ancient
TEs, we manually checked and listed the eleven TEs, which exist in at least two species including mouse (Table 1). No TE was found in opossum and chicken, possibly because of the low homology between this two species and mouse.

In Pldi exon 1 to 3 and $A k 158810$ exon 1 to 4 , no ancient TE was detected. However, almost half of all the defined ancient TEs locate in Ak158810 exon 5 (Table 2 \& Figure 4). Inside the longest Pldi intron 1, four ancient TEs were identified, three of which also locate in the overlapped $A k 158810$ intron region (Table 2 \& Figure 4). 


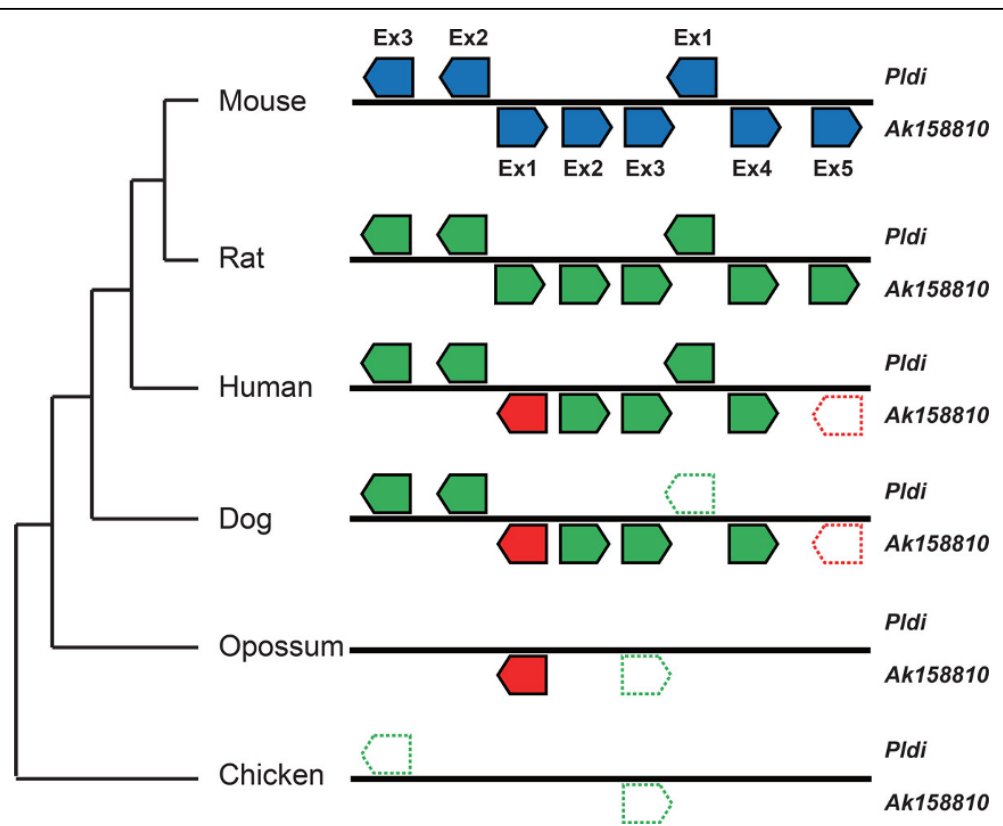

Figure 5 Formation of Pldi-Ak158810 exon sequences. Green pentagons represent for homologue sequences match with specific mouse exons, red ones represent for inversed exons. Pentagons in bold lines suggest homologues with low similarity or low percent of coverage.

The data shows no evidence that insertions of TEs have been involved in most of the exons at the loci during recent period of time, except for $A k 158810$ exon 5. The formation of the last exon of $A k 158810$ and intron sequences of both Pldi and Ak158810 are associated with various types of transposable events.

\section{An inverse element is highly conserved and obtains a} reduced substitution rate after rearrangement

From genomic sequence in mammals, we noticed the Pldi-Ak158810 loci was interrupted by chromosome rearrangement in a period of time before its emergence

Table 1 Transposable elements (TEs) that contributed to the formation of ancestral PIdi-Ak158810 sequences.

\begin{tabular}{llll}
\hline TE symbol & TE name & Repeat Class & Species with the TE \\
\hline A & MIR & SINE & mouse, rat, human, dog \\
B & MIR & SINE & mouse, rat, human, dog \\
C & Chap1_Mam & DNA & mouse, human \\
D & MER91A & DNA & mouse, rat, human, dog \\
E & URR1B & DNA & mouse, rat \\
F & MTEb & LTR & mouse, rat \\
G & MIR3 & SINE & mouse, rat, human \\
H & MT2B2 & LTR & mouse, rat \\
I & B1F & SINE & mouse, rat \\
J & PB1D10 & SINE & mouse, rat \\
K & L1MD3 & LINE & mouse, human, dog \\
\hline
\end{tabular}

Only TEs in at least 2 species including mouse are listed. SINE, short interspersed nuclear elements; LINE, long interspersed nuclear elements; DNA, DNA repeat elements; LTR, long terminal repeat elements, including retroposons. in mouse lineage. Interestingly, one of the rearranged fragments associated with Ak158810 exon 1 is highly conserved among species. From this point of view, we estimated the substitution rate of this highly conserved region among species to test whether inversion contribute to fixation of local region. To better learn the evolution of this loci, we examined the change in substitution rate during the fixation of 4 species, mouse, rat, human and dog. Taking the exon sequences of flanking genes ( $p c b d 1$, slc29a3, sgpl1, unc5b) as a reference, we constructed a simple model to test the rate change at two time points: the point of chromosome

Table 2 Gene composition originated from TE

\begin{tabular}{lll}
\hline Gene & Element & Origin from TE \\
\hline Pldi & exon 1 & \\
& exon 2 & \\
& exon 3 & \\
& intron 1 & A, B, C, D \\
& intron 2 & \\
Ak158810 & exon 1 & \\
& exon 2 & \\
& exon 3 & \\
& exon 4 & \\
& exon 5 & G, H, I, I, K \\
& intron 1 & B, C \\
& intron 2 & D \\
& intron 3 & \\
& intron 4 & E, F \\
\hline
\end{tabular}

$A, B, C, D, E, F, G, H, I, J, K$ represent different TE used in Table 1. 
Table 3 A simplified model to test the change of substitution rate at two time point: occurrence of the inversion and emergence of PIdi and Ak158810 gene.

\begin{tabular}{lllll}
\hline Reference & Test sequence & raverHD & raverHR & raverMR \\
\hline Exons of surrounding genes & Conserved element 1 (inversed region) & 2.5971 & 2.0603 & 1.406 \\
Exons of surrounding genes & Conserved element 2 & 1.6694 & 2.1832 & 1.5047 \\
\hline
\end{tabular}

Normalized average substitution rates during specific lineage (e.g. "HD" stands for human and dog) are calculated.

rearrangement and the emergence of Pldi and Ak158810 in mouse lineage (Figure 5). We extracted sequences of two conserved elements (CE1, conserved element 1 in the inversion; CE2, conserved element 2 near Ak158810 exon 3 ), which could be detected by Blastn in distant organisms.

Compared with surrounding genes (Additional File 7 \& Table 3), both CE1 and CE2 obtain the lowest normalized rates during mouse-rat divergence, in line with result from a previous study that purifying selection is acting on Pldi region after its emergence [10]. Furthermore, for $\mathrm{CE} 1$, the average rate of human-dog divergence is higher than that of human-rat, which implies the substitution rate of this element was slightly reduced after rearrangement. For CE2, not involved in rearranged regions, the tendency is opposite. The data shows the possibility that the specific elements of the Pldi-Ak158810 loci established their fixation at an early time before the gene birth. Inversion of CE1 may contribute to its acquisition of purifying selection, causing a slightly reduced substitution rate.

\section{Discussion}

Various factors contribute to the formation of PIdiAk158810 sequence

A new lncRNA gene could emerge through different scenarios, such as metamorphosis from a protein-coding gene, interrupted by tandem repeat and transposable elements, and de novo origin from an intergenic region. Our analysis further confirmed the inter-genic origin of Pldi$A k 158810$ sequence without any clues of long genomic duplication in a recent past. Tracing back in history, both transposable events and chromosome rearrangements were found in the region. In conclusion, the formation of the Pldi-Ak158810 loci, which became a pair of lncRNA genes in mouse lineage, was affected by multiple factors.

\section{Fixation of partial Pldi-Ak158810 sequence before gene birth}

A previous study indicates that the conservation of noncoding RNA is only slightly higher than that of intergenic region [10]. In Pldi region, reduced polymorphism has been detected in specific mouse lineage, which suggests the present of purifying selection. Nevertheless, we found in our data that partial Pldi-Ak158810 sequence is conserved in all mammalians. It raises the possibility that purifying selection may be acquired in partial Pldi$A k 158810$ region much earlier than the gene birth.

We checked factors that could be responsible to the early fixation. Our calculation of substitution rate change shows that the inversed $A k 158810$ exon 1 was prone to decreasing the evolutional ability after inversion event, relative to surrounding genes (Table 3 \& Additional File 7). This trend may represent for an increasing natural selection [25-27]. We also checked DNA modification of the region in human. A series significant signals of demethylation in CpGs are highly correlated with the conserved inversed element, CE1 (Figure 6 \& Additional File 3) using Encode browser [28], CE1 is overlapped with the promoter region of Pldi 's antisense gene, $A k 158810$, and the promoter sequence in mouse was found with low DNA methylations [21]. Furthermore, from the transcription factor binding site conserved tracks in UCSC, we find this CE1 homologue site is a potential transcription factor binding site of Chx10 conserved in both human and mouse (Figure 6) [24]. This binding site exists both in human and mouse.

According to these observations, we suggested in species other than mouse, partial region of Pldi-Ak158810 loci could not be simply recognized as "non-functional" before the birth of Pldi.

\section{Birth order of $A K 158810$ and PIdi}

It has been known that two neighboring genes may form a transcriptional unit [11], which is correlated with expression. As for this case, we assumed the earlier developed lncRNA might influence the birth of the other one by expression level. We attempted to detect the birth order of $A K 158810$ and Pldi, According to previous studies, the birth order of $A k 158810$ and Pldi may not quite clear for the following reasons: first, testis where Pldi was born has been considered as an important organ for the emergence of a novel gene $[4,29]$. According to RNA-seq data (CSHL) and previous study [10], Pldi is a testis-specific lncRNA, while $A k 158810$ is likely to have a wide expression range, such as heart, spleen and kidney (Additional File 8). That indicates that $A k 158810$ seems to be a not that young gene as Pldi [4,30]; Second, considering northern blot experiment, Pldi exists in more species or lineages in mouse testis $[10,31]$, inferring that it is more likely to be older 


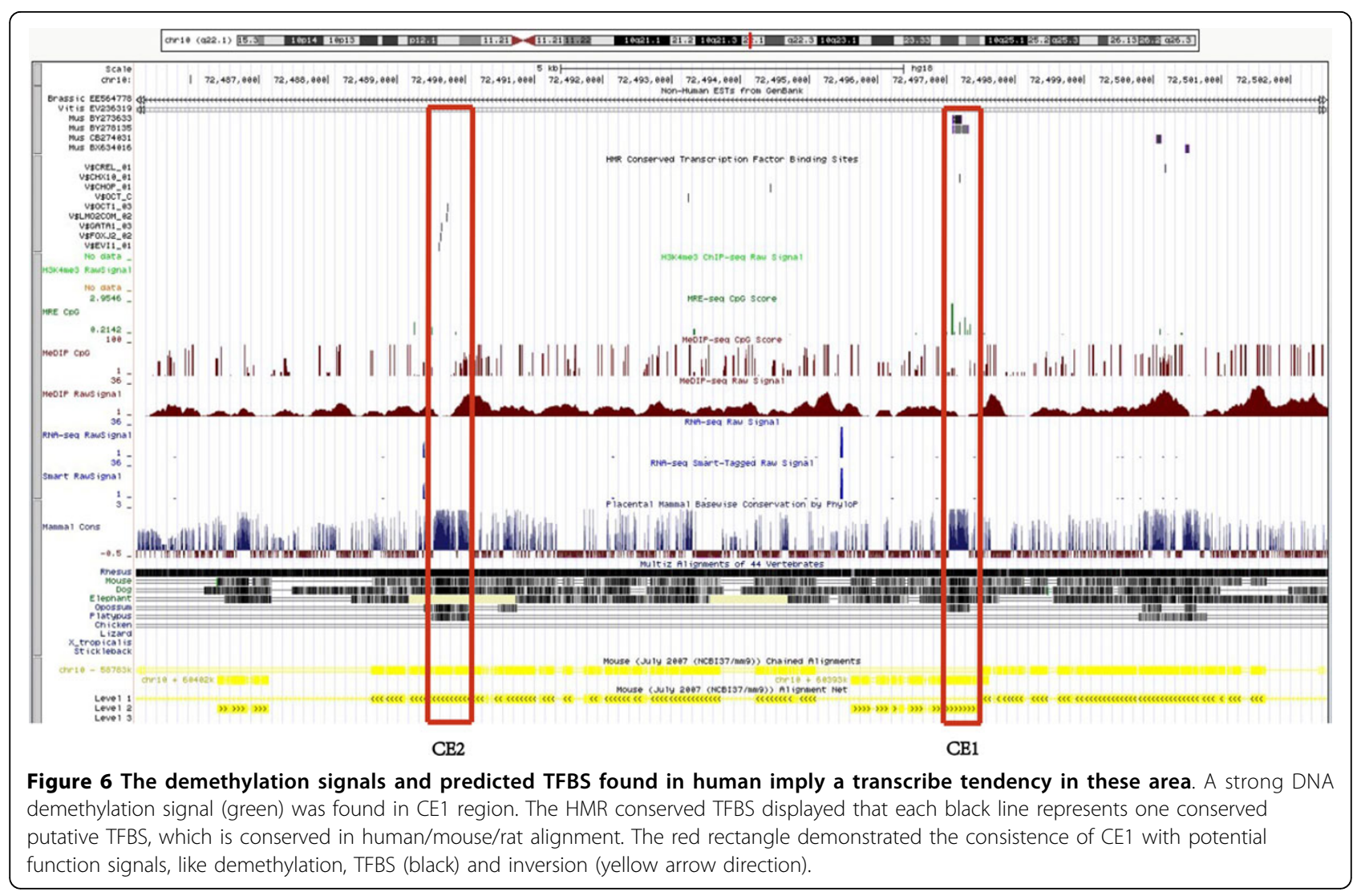

than Ak158810. The conflict result, together with the phenomenon, that the expressions of both lncRNAs are limited in mouse, demonstrated that AK158810 and Pldi were newly transcribed lncRNAs in a similar age after the divergence of mouse and rat.

\section{Conclusion}

In this study, we comprehensively analyzed the sequence origin of a lncRNA antisense gene pair, Pldi-Ak158810. We found out various factors, including rearrangement, transposable elements, contributed to the formation of the sequence. We also figured out partial sequence of the entire loci is highly conserved in mammalians before the birth and provided evidences and correlated factors for the early fixation of conserved elements.

\section{Lists of abbreviation}

Pldi: Polymorphic derived intron-containing; lncRNA: long non-coding RNA; ESTs: expressed sequence tags; TEs: Transposable elements; CE1: Conserved Element 1; CE2: Conserved Element 2; SINE: Short interspersed nuclear elements; LINE: Long interspersed nuclear elements; DNA: DNA repeat elements; LTR: Long terminal repeat elements, which include retroposons; TFBS: Transcriptional factor binding site; CSHL: Cold Spring Harbor Lab; HMR: Human, mouse, rat.

\section{Additional material}

Additional file 1: PIdi and its antisense transcript Ak158810. It's a screenshot of the region contains Pldi and Ak158810 from UCSC Browser. These two transcripts share about 8000 bps long. From EST data, there is a potential antisense region overlapped between first exon of Pldi and fourth exon of Ak158810.

Additional file 2: The subject genomes for Blastn were taken from UCSC. The genomes of 13 vertebrates were downloaded from UCSC. And the versions of the genome were listed according to the species.

Additional file 3: The Methylation degree of CpGs in Pldi-Ak158810 region. This data was obtained from the forebrain tissue of a lab mouse (GSM809309). The methylation score in y-axis represents the possibility of a CpG site methylated. The $x$-axis represents the genome position. The arrow showed the direction of the transcript. CpG sites are enriched in the first exon of Ak158810 in CE1 region. Pldi contains few CpG sites near transcript start region. The blue arrow shows the direction of the transcription.

Additional file 4: The splicing evidence for transcription Ak158810. We Compared Ak158810, including its introns with mouse EST database in NCBI. Several tags could be mapped to Ak158810 exons (in blue cycles). And the first splicing site between exon 1 and exon 2 could be observed.

Additional file 5: Potential ORF of $\boldsymbol{A K} \mathbf{1 5 8 8 1 0 .} \mathrm{ORF}$ finder demonstrated the potential ORF and its position in the transcript of Ak158810. Green frame represents the potential ORF. Only one potential open reading frames longer than 100 amino acids. And two AUG codons with shorter reading frames (about 70 amino acids) precede this long ORF. Frame site, position and length were demonstrated aside.

Additional file 6: Blastn result for PIdi-Ak158810 region in 13 species. Sequence in Pldi-Ak158810 region is used as an inquiry to detect the homologue sequence in 12 other vertebrates by Blastn. The 
command for Blastn is 'blastn - $\mathrm{db} \$ \mathrm{db}$-task blastn - $\mathrm{db} \$ \mathrm{db}$-task megablast -query \$query -out \$out -outfmt 6'. The length of initial exact match is at least bigger than 28. 'db' is the reference genome of 12 species. 'query' is the sequence of Pldi and Ak158810 loci in mouse.

\section{Additional file 7: Sequence alignment file of $\mathbf{4}$ key species.}

Alignment files contained the regions we picked from the multiz file of 30 vertebrates to do alignment among dog, human, mouse and rat to calculate the substitution rate in Table 3 based on the model raised in the Figure 1. The alignment file includes inversed element (Conserved element 1), tandem elements (Conserved element 2) and surrounding genes.

Additional file 8: Rna-seq in different tissues of Mouse show this PIdi and Ak158810 loci is a dynamic transcriptional state in different tissues. Long RNA-seq data from Encode CSHL provided the expression level of PIdi-Ak158810 region in different tissues of mouse from UCSC Browser. wide expression signals of Pldi and Ak158810 were found in testis. In heart, kidney and spleen, similar transcripts in region of Ak158810 were enriched. Other tissues did not show specific expression of these two transcripts.

\section{Competing interests}

The authors declare that they have no competing interests.

\section{Authors' contributions}

YL.D., SD.L., X.D., H.S., C.L., Z.L. and BL.Y. carried out all the analysis in this study. X.D. conceived of the study. YL.D., SD.L., X.D., GH.D. and YX.L. wrote the manuscript. All authors read and approved the final manuscript.

\section{Acknowledgements}

We thank Prof. Lei Liu, Dr. Guangyong Zheng, Dr. Zhen Wang, Jie Wang, and Tiancheng Liu, for their helpful comments and suggestions. This work was supported by the National Key Basic Research Program of China, known as 973 Program (grant number: 2011CB910204, grant number: 2010CB529206, grant number: 2010CB912702), the Research Program of The Chinese Academy of Sciences (grant number: KSCX2-EW-R-04, grant number: KSCX2YW-R-190, grant number: 2011KIP204) and National Natural Science Foundation of China (grant number: 31070752).

\section{Declarations}

Funding for the publication fee comes from National Natural Science Foundation of China (grant number: 31070752).

This article has been published as part of BMC Genomics Volume 14 Supplement 8, 2013: Selected articles from the International Conference on Intelligent Biology and Medicine (ICIBM 2013): Genomics. The full contents of the supplement are available online at http://www.biomedcentral.com/ bmcgenomics/supplements/14/S8.

\section{Authors' details}

${ }^{1}$ Key Laboratory of Systems Biology, Shanghai Institutes for Biological Sciences, Chinese Academy of Sciences, 320 Yueyang Rd. Shanghai 200031, PR China. ${ }^{2}$ Graduate School of Chinese Academy of Sciences, 19 Yuquan Rd. Beijing 100049, PR China. ${ }^{3}$ Shanghai Center for Bioinformation Technology, 1278 Keyuan Rd. Shanghai 201203, PR China. ${ }^{4}$ School of Life Sciences, Fudan University, 220 Handan Rd. Shanghai 200433, PR China.

\section{Published: 9 December 2013}

\section{References}

1. Ponting $C P$, Oliver $P L$, et al: Evolution and functions of long noncoding RNAs. Cell 2009, 136(4):629-41.

2. Mercer TR, Dinger ME, et al: Long non-coding RNAs: insights into functions. Nat Rev Genet 2009, 10(3):155-9.

3. Tautz D, Domazet-Loso T: The evolutionary origin of orphan genes. Nat Rev Genet 2011, 12(10):692-702.

4. Kaessmann $\mathrm{H}$ : Origins, evolution, and phenotypic impact of new genes. Genome Res 2010, 20(10):1313-26.
5. Duret L, Chureau C, et al: The Xist RNA gene evolved in eutherians by pseudogenization of a protein-coding gene. Science 2006, 312(5780):1653-5.

6. He S, Liu S, et al: The sequence, structure and evolutionary features of HOTAIR in mammals. BMC Evol Biol 2011, 11:102.

7. Cai J, Zhao R, Jiang H, Wang W: De novo origination of a new proteincoding gene in Saccharomyces cerevisiae. Genetics 2008, 179(1):487-496.

8. Carvunis AR, Rolland T, Wapinski I, Calderwood MA, et al: Proto-genes and de novo gene birth. Nature 2012, 487(7407):370-4.

9. Derrien $T$, Johnson $R$, et al: The GENCODE v7 catalog of human long noncoding RNAs: analysis of their gene structure, evolution, and expression. Genome Res 2012, 22(9):1775-89.

10. Heinen TJ, Staubach $F$, et al: Emergence of a new gene from an intergenic region. Curr Biol 2009, 19(18):1527-31.

11. Katayama S, Tomaru $Y$, Kasukawa $T$, et al: Antisense transcription in the mammalian transcriptome. Science 2005, 309(5740):1564-6.

12. Engström PG, Suzuki $H$, Ninomiya $N$, et al: Complex Loci in human and mouse genomes. PLoS genetics 2006, 2(4):e47.

13. Dinger ME, Amaral PP, Mercer TR, et al: Long noncoding RNAs in mouse embryonic stem cell pluripotency and differentiation. Genome Research 2008, 18(9):1433-45.

14. Wheeler DL, Church DM, Federhen S, et al: Database resources of the National Center for Biotechnology. Nucleic Acids Res 2013 31(1):28-33

15. Thompson JD, Gibson T, et al: Multiple sequence alignment using ClustalW and ClustalX. Curr Protoc Bioinformatics 2002, Chapter 2: Unit 2.3.

16. Saitou N, Nei M: The neighbor-joining method: a new method for reconstructing phylogenetic trees. Molecular Biology and Evolution 1987, 4(4):406-425.

17. Blanchette $M$, Kent WJ, et al: Aligning multiple genomic sequences with the threaded blockset aligner. Genome Res 2004, 14(4):708-15.

18. Sakuraba $Y$, Kimura $T$, et al: Identification and characterization of new long conserved noncoding sequences in vertebrates. Mamm Genome 2008, 19(10-12):703-12

19. Yang Z: PAML: a program package for phylogenetic analysis by maximum likelihood. Comput Appl Biosci 1997, 13(5):555-6.

20. Smit AFA, Hubley R, Green P: RepeatMasker Open-3.0. 1996 [http://www repeatmasker.org]

21. Xin $Y, O^{\prime}$ Donnell AH, Ge $Y$, Chanrion B, et al: Role of CpG context and content in evolutionary signatures of brain DNA methylation. Epigenetics 2011, 6(11):1308-18.

22. Maunakea AK, Nagarajan RP, et al: Conserved role of intragenic DNA methylation in regulating alternative promoters. Nature 2010, 466(7303):253-7.

23. Parkhomchuk D, Borodina T, Amstislavskiy V, Banaru M, Hallen L, Krobitsch S, Lehrach $\mathrm{H}$, Soldatov A: Transcriptome analysis by strandspecific sequencing of complementary DNA. Nucleic Acids Res 2009, 37(18):e123.

24. Wingender $\mathrm{E}$ : The TRANSFAC project as an example of framework technology that supports the analysis of genomic regulation. Briefings in bioinformatics 2008, 9(4):326-332.

25. Lippman Z, Gendrel A V, Black M, et al: Role of transposable elements in heterochromatin and epigenetic control. Nature 2004, 430(6998):471-476.

26. Prabhakar S, Noonan JP, Pääbo S, Rubin EM: Accelerated evolution of conserved noncoding sequences in humans. Science 2006 314(5800):786.

27. Katzman Sol, Kern AD, Bejerano G, Fewell G, Fulton RK, Wilson, Salama SR, Haussler, David: Human Genome Ultraconserved Elements are Ultraselected. Science 2007, 317(5840):915.

28. Rosenbloom KR, Dreszer TR, Pheasant M, et al: ENCODE whole-genome data in the UCSC Genome Browser. Nucleic Acids Res 2012, 40(Database issue):D912-7.

29. Marques AC, Dupanloup I, Vinckenbosch N, et al: Emergence of young human genes after a burst of retroposition in primates. PLOS biology 2005, 3(11):e357.

30. Long $M$, Betran $E$, et al: The origin of new genes: glimpses from the young and old. Nat Rev Genet 2003, 4(11):865-75. 
31. Heinen $T$ J , Staubach $F$, et al: Emergence of a new gene from an intergenic region. Curr Biol 2009, 19(18):1527-31, (Figure S8).

32. Margulies EH, Maduro WB, Thomas PJ, et al: Comparative sequencing provides insights about the structure and conservation of marsupial and monotreme genomes. Proceedings of the National Academy of Sciences of the United States of America 2005, 102(9):3354-3359.

\section{doi:10.1186/1471-2164-14-S8-S6}

Cite this article as: Dai et al:: The de novo sequence origin of two long non-coding genes from an inter-genic region. BMC Genomics 201314

(Suppl 8):S6.

Submit your next manuscript to BioMed Central and take full advantage of:

- Convenient online submission

- Thorough peer review

- No space constraints or color figure charges

- Immediate publication on acceptance

- Inclusion in PubMed, CAS, Scopus and Google Scholar

- Research which is freely available for redistribution

Submit your manuscript at www.biomedcentral.com/submit
C Biomed Central 